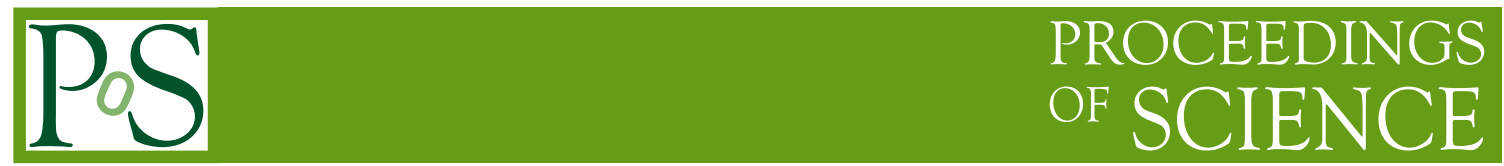

\title{
Experimental results from MAMI
}

\section{Harald Merkel*}

Johannes Gutenberg University

E-mail: merkelekph.uni-mainz.de

Experimental tests of predictions of Chiral Perturbation Theory are traditionally a major topic of the experimental program at the Mainz Microtron (MAMI). In this summary, recent results and an update on the current program on $\eta$ decays, polarizabilities of the nucleon, and threshold pion production is presented.

6th International Workshop on Chiral Dynamics

July 6-10 2009

Bern, Switzerland

\footnotetext{
${ }^{*}$ Speaker.
} 


\section{Introduction}

The strength of the Mainz Microtron is the ability to perform precision experiments in the energy regime below $1.5 \mathrm{GeV}$. This makes the machine an ideal tool to study effects of Chiral Dynamics, which manifests themself usually at lowest energies, e.g. in the threshold region of the production of pions, which are the Goldstone Bosons of the chiral symmetry breaking.

Meanwhile, chiral dynamics play a crucial role in the analysis of nearly all experiments in the energy range of MAMI. In this summary, only results on a few selected recent experiments will be presented, which have their main focus on testing chiral dynamics.

\section{2. $\eta$ and $\eta^{\prime}$ Decays}

With the energy upgrade to $1.5 \mathrm{GeV}$ of MAMI in the year 2007, the high luminosity of MAMI could be utilized for the production of $\eta$ and $\eta^{\prime}$ mesons with high statistics for the study of their hadronic decay.

The decay of the $\eta$ and $\eta^{\prime}$ meson into three neutral pions is forbidden by exact isospin symmetry. Thus, this decay is in first order proportional to the explicit symmetry breaking introduced by the light quark mass difference $m_{d}-m_{u}$, providing an ideal testing ground for Chiral Perturbation Theory.

A first calculation of the decay amplitude $A(s, t, u)$ to order $\mathscr{O}\left(p^{2}\right)$ was done by Gasser and Leutwyler in 1985 [1], leading to the result

$$
A(s, t, u)=\frac{B_{0}\left(m_{u}-m_{d}\right)}{3 \sqrt{3} F_{\pi}^{2}}\left[1+\frac{3\left(s-s_{0}\right)}{M_{\eta}^{2}-M_{\pi}^{2}}\right] \sim \frac{m_{d}-m_{u}}{m_{s}-\hat{m}}
$$

It turns out, that up to now even calculations up to order $\mathscr{O}\left(p^{6}\right)$ are not sufficient to describe the experimental data.

For the experiments, the size of the statistical sample is usually the limit for the accuracy. Besides a high current and high duty cycle accelerator, a detector with nearly hermetic coverage of the solid angle for the detection of the photons of the $\pi^{0} \rightarrow \gamma \gamma$ decay is mandatory. In Mainz, the Crystal Ball detector at the tagged photon beam of the A2 Collaboration is used for these experiments, which has as central part a photon detector of $672 \mathrm{NaJ}$ crystals covering the angular range from $20^{\circ}$ up to $160^{\circ}$. In forward direction, a detector with $510 \mathrm{BaF}_{2}$ crystals covers the region below $20^{\circ}$.

A sample of $3 \cdot 10^{6}$ detected $\eta$ decays could be accumulated up to now. The experimental observable to access the decay amplitude $A$ is the slope parameter $\alpha$, which is defined as

$$
\begin{aligned}
\left|A_{\eta \rightarrow 3 \pi^{0}}\right|^{2} & \sim 1+2 \alpha z+\cdots \\
\text { with } \quad z & =6 \sum_{i=1}^{3} \frac{\left(E_{i}-m_{\eta} / 3\right)^{2}}{\left(m_{\eta}-3 m_{\pi^{0}}\right)^{2}}=\frac{\rho^{2}}{\rho_{\max }^{2}} .
\end{aligned}
$$

In addition, a cusp can be detected at the threshold for the $\pi^{0} \pi^{0} \rightarrow \pi^{+} \pi^{-}$rescattering amplitude.

A first experiment was performed with MAMI-B [2] before the energy upgrade and determined a slope parameter of

$$
\alpha=-0.0319 \pm 0.0015 \pm 0.0016 \text {. }
$$


The continuation of this experiment with MAMI-C [3] yield the result

$$
\alpha=-0.0322 \pm 0.0012 \pm 0.0022 \text {. }
$$

These values for the slope parameter are consistent with previous experiments at Brookhaven and KLOE, but are still in clear discrepancy to the results of pure Chiral Perturbation Theory.

A calculation using a unitary approach[4] could show, that in this approach the data could be fitted to agree with Chiral Perturbation Theory. This is, however, not yet a final confirmation of the CHPT results. For a detailed discussion including the cusp effect of the slope parameter see the contribution of S. Prakov to this conference. In the near future, the accumulated statistical sample will be increased by nearly an order of magnitude.

\section{Polarizabilities of the Nucleon}

Polarizabilities are defined as the response of a system to an external field. The ratio of the induced electric or magnetic dipole moment to the applied external electromagnetic field is defined as electric polarizability $\alpha$ or magnetic polarizability $\beta$, respectively. The polarizabilities are a direct measure of the stiffness of the nucleon and are a fundamental property of the nucleon in the static limit and can be calculated in Chiral Perturbation Theory. Including the spin of the nucleon and the probing virtual photon of the electromagnetic field, spin polarizabilities can be defined in addition[5].

\subsection{Real Compton Scattering}

The polarizabilities can be determined by Compton Scattering. For real Compton Scattering, they appear in first order as the deviation of the cross section from the point-like case

$$
\frac{d \sigma}{d \Omega}=\frac{d \sigma_{0}}{d \Omega}-\frac{e^{2}}{4 \pi m_{p}}\left(\frac{q^{\prime}}{q}\right)^{2} q q^{\prime}\left\{\frac{1}{2}(\bar{\alpha}+\bar{\beta})(1+\cos \theta)^{2}+\frac{1}{2}(\bar{\alpha}-\bar{\beta})(1-\cos \theta)^{2}\right\}+\cdots
$$

In forward direction, the sum $\alpha+\beta$ can be extracted, while in backward direction the difference can be extracted. The electric and magnetic polarizabilities were determined at MAMI[6] to

$$
\begin{aligned}
& \alpha=\left(12.1 \pm 0.3_{\text {stat }} \pm 0.5_{\text {syst }}\right) 10^{-4} \mathrm{fm}^{3} \\
& \beta=\left(1.6 \pm 0.4_{\text {stat }} \pm 0.6_{\text {syst }}\right) 10^{-4} \mathrm{fm}^{3}
\end{aligned}
$$

This result was within the error bar in good agreement with calculations in ChPT. The next step is, to determine the four spin polarizabilities. For this, a proposal at MAMI was submitted[7], to measure several beam-target double-polarization observables to extract all spin polarizabilities.

\subsection{Virtual Compton Scattering}

The polarizabilities can be generalized to the case of finite incoming photon virtuality $Q^{2}$. These so called generalized polarizabilities are related to the local distribution of the polarizabilities or can be interpreted as "form factor" measurement in an external field. By multiple decomposition 10 generalized polarizabilities can be defined in the static limit[8], and it was shown in ref. [9], that only six of these are independent. 


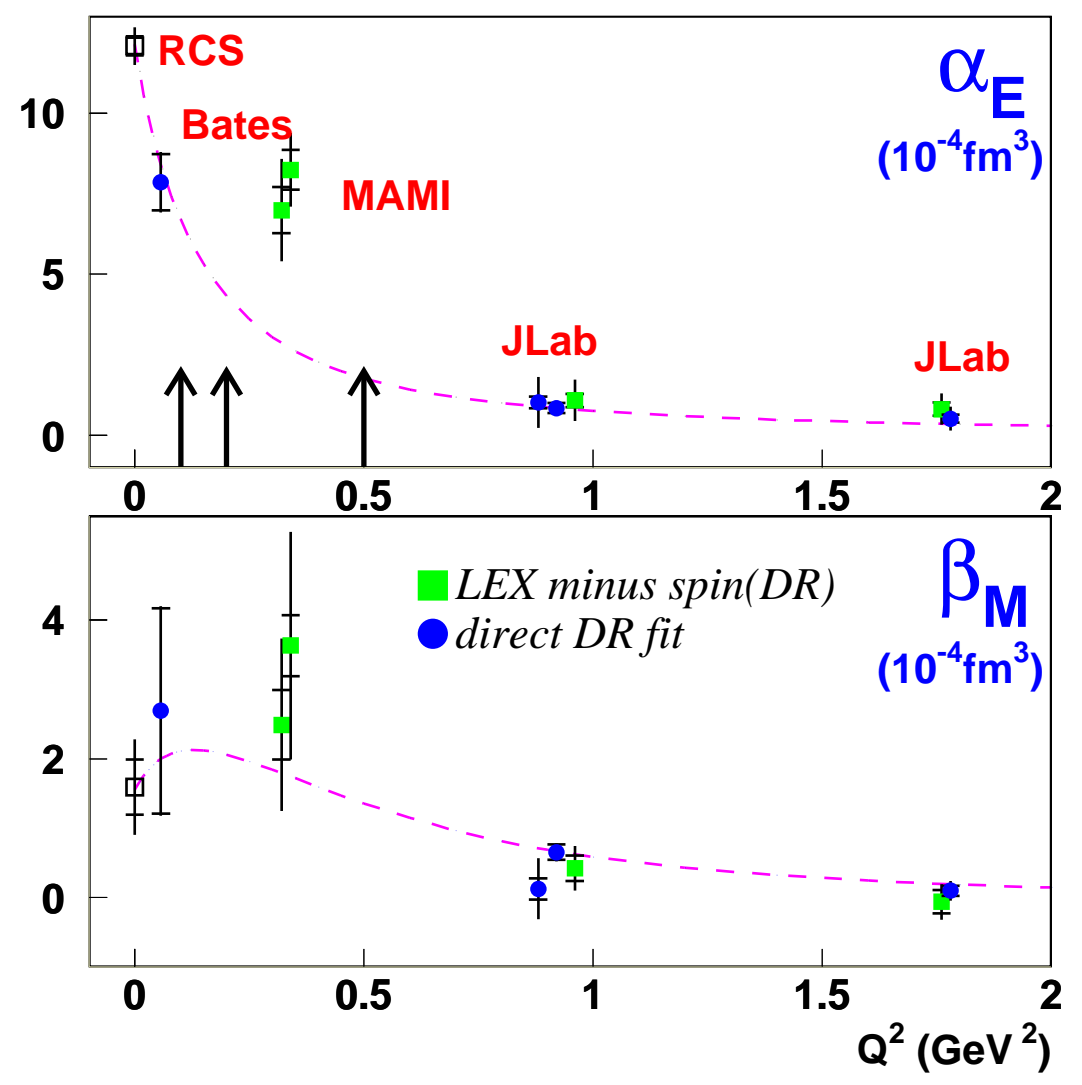

Figure 1: Results on $\alpha$ and beta from unpolarized Virtual Compton Scattering. The dashed line shows the result of a DR calculation. The three arrows denote the $Q^{2}$ values of a planned experiment at MAMI.

The cross section of Virtual Compton Scattering is overwhelmed by the background of radiative processes, i.e. elastic scattering with the emission of a photon from incoming or outgoing electron (Bethe-Heitler process) or from the incoming or outgoing proton (Born-Term). The polarizabilities contribute as the interference between the VCS process and the Bethe-Heitler and Born process. At the moment, two different approaches are used to extract the polarizabilities from the cross section. The Low Energy Expansion (LEX) uses the expansion in outgoing photon momenta, which is proportional to the effects of the polarizabilities in the limit to vanishing photon momentum. A second approach based on Dispersion Relations[10] uses the input of pion production data for a more detailed modeling of the energy dependence of VCS and is in principle not limited to low energies.

Several unpolarized experiments were performed at MAMI, MIT-Bates, and JLab at several different values of $Q^{2}$. Fig. 1 summarizes the results of these experiments on the non-spin polarizabilities $\alpha$ and $\beta$. The lines show the results of the dispersion analysis with the ad hoc assumption of a dipole shape of the $Q^{2}$ dependence of the polarizabilities. This arbitrary assumption holds well for the Bates and JLab results, but is in clear disagreement with the MAMI results, which were confirmed with a second measurement. The arrows denote the $Q^{2}$ values of a planned experiment[11] at MAMI to clarify the $Q^{2}$ dependence of the polarizabilities. 


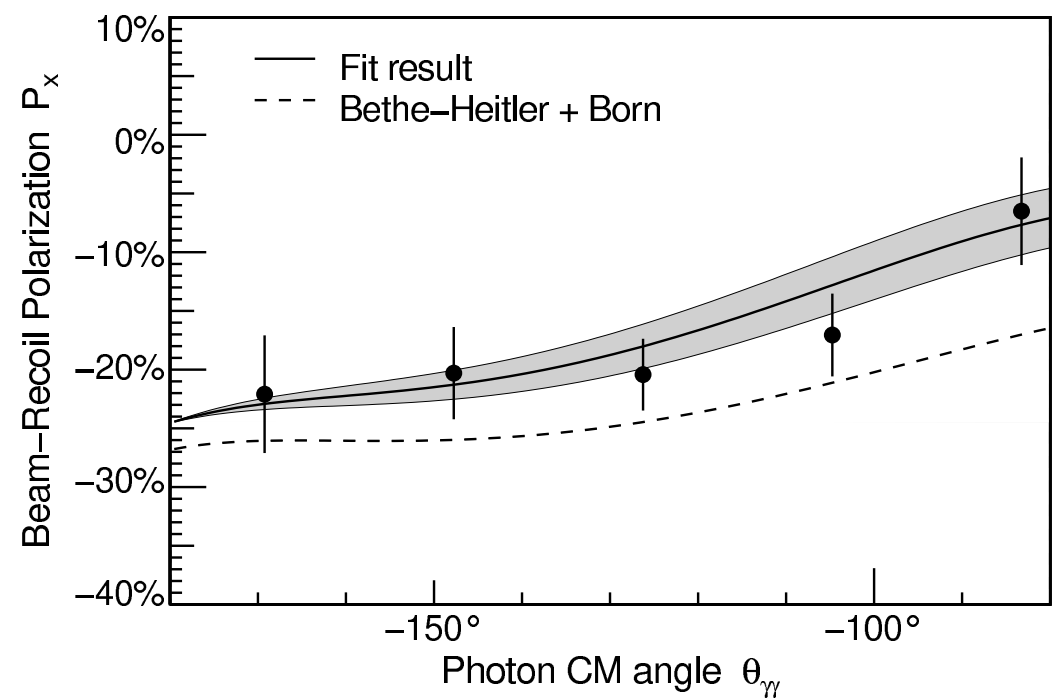

Figure 2: Beam-Recoil double polarization observable $P_{x}$ (Preliminary!). The dashed line shows the contribution of the Bethe-Heitler-Effect, the solid line the fit including polarizabilities.

\subsection{Double Polarization Observables}

To extract spin polarizabilities, again double polarization observables are necessary. At the A1 Collaboration at MAMI, a first experiment to determine the beam-recoil observables were performed. For this, a polarimeter was used in the focal plane of a magnetic spectrometer to determine the focal plane polarization of the recoil proton. By a maximum-likelihood-method the center-ofmass polarizations could be determined. Fig. 2 shows the results of this measurement[12]. The clear effect of the polarizabilities is visible, however the main contribution to this effect is due to the non-spin-polarizabilities. The spin-polarizabilities turned out to be smaller than expected.

\section{Threshold Pion Production}

The direct threshold production of the Goldstone Bosons of the chiral symmetry via electroand photoproduction is a well known testing ground for the convergence radius of Chiral Perturbation Theory including heavy baryons. While the charged pion production is dominated by the Kroll-Ruderman-Term, the neutral pion production is a stringent test of ChPT.

Close to threshold, the production amplitude is dominated only by $s$ - and $p$-waves, so only a limited number of observables have to be measured for a complete description of the production amplitude. In addition, the energy dependence of the multipoles is known: the $s$-wave amplitude of $\pi^{0} p$ production shows a clean cusp effect by the opening $\pi^{+} n$ threshold, the $\mathrm{p}$-waves are real and roughly proportional to the outgoing pion momentum. Thus, the threshold photoproduction is completely determined by only four real numbers.

Despite the simplicity of the structure of the amplitudes, the experiments are still challenging since cross sections are small close to threshold. A series of experiments on photoproduction showed nevertheless an impressive agreement between experiments and calculations in the framework of Heavy Baryon Chiral Perturbation Theory. 

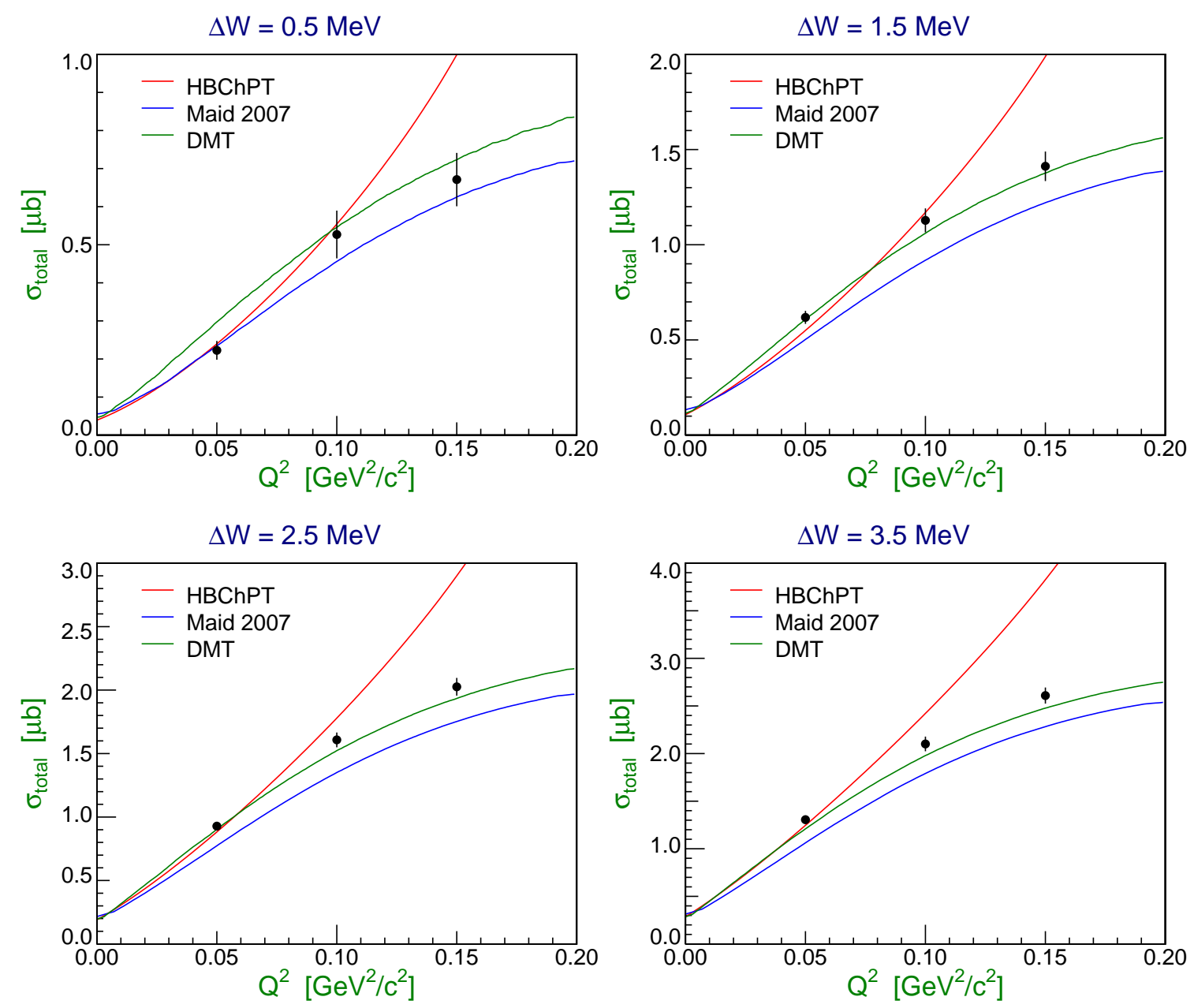

Figure 3: $Q^{2}$ dependence of the cross section of threshold pion electroproduction. The red line shows a calculation in HBChPT[16], the blue line the phenomenological MAID model[17], and the green line the DMT model[18]. The deviation of the HBChPT line is meaningless, since this calculation was fitted to a data set with large systematic errors.

The situation is different for electroproduction. Compared to photo production, the multipoles are now functions of the photon virtuality $Q^{2}$ and additional longitudinal multipoles appear. The convergence radius of HBChPT in $Q^{2}$ is a priory not known and has to be determined by experiment. Experimentally, the background conditions of electroproduction and the need for a separation of longitudinal and transverse contribution introduces additional systematic errors.

First experiments in NIKHEF [13] and MAMI [14, 15] were not conclusive. While the experiments showed rough agreement with HBChPT calculations [16] when interpreted in terms of multipoles, the inconsistency between the data sets was shown most prominent when comparing the $Q^{2}$ evolution of the total cross section. The obvious problem of the systematic errors lead to the design of an experiment with emphasis on a significantly reduced systematic error to provide a consistent data set over the interesting $Q^{2}$ range.

Fig. 3 shows the preliminary result of this experiment in comparison with some models. No LT-separation was performed for this figure, since this would enhance the systematic errors. At 
three $Q^{2}$ values, differential cross sections were taken for four different energy bins below $\pi^{+} n$ threshold. As can be seen, the consistency of the data set improved and shows now the expected $Q^{2}$ behavior. For comparison, three models are included in the figure. The MAID model[17] is a phenomenological model, including all pion production data. This model is dominated by photoproduction data and data at higher $Q^{2}$ values. The remaining discrepancy in total cross section can probably be removed by including the new data in the fit. The DMT model[18] generates the threshold region dynamically and is in good agreement. The discrepancy of the HBChPT model results only from the fact, that this model was fitted to the elder inconsistent data sets only up to $Q^{2}=0.10 \mathrm{GeV}^{2} / c^{2}$. A refit including the data points $Q^{2}=0.15 \mathrm{GeV}^{2} / c^{2}$ would show a better agreement with the data also at higher $Q^{2}$ values.

\subsection{Future experiments on threshold meson production}

At MAMI, several experiments on further threshold production experiments are planed. In photoproduction, the polarized beam asymmetry $\Sigma$ is needed to separate the $p$-wave combination $P_{2}^{2}+P_{3}^{2}$. The existing data on this observables were limited in statistics and allowed only for a single energy bin. New data on this observable were taken with the Crystal Ball Detector and are under analysis.

While the real part of the $s$ wave amplitude is measured in the cross section, the transverse polarized target asymmetry is sensitive to the imaginary part of this amplitude. This provides an access to the charge exchange scattering length. In the same experiment, using a polarized photon beam, the beam-target-asymmetry $F$ can be used to explore the $d$ wave contribution to the cross section, which turned out to be not negligible in the considered energy range.

Finally, the experimental program on pion production will be extended to the $S U$ (3) sector by threshold kaon production.

\section{References}

[1] J. Gasser, H. Leutwyler, Nucl. Phys. B 250, 539 (1985).

[2] M. Unverzagt et al., Eur. Phys. J. A39, 169-177 (2009).

[3] S. Prakhov et al., Phys. Rev. C79, 035204 (2009).

[4] B. Borasoya and R. Nißler, Eur. Phys. J. A 26, 383-398 (2005).

[5] S. Ragusa, Phys. Rev. D 47, 3757 (1993).

[6] V. Olmos de Leon et al., EPJ A 10 207-215 (2001).

[7] MAMI Proposal A2-11/09, D. Hornidge, E.J. Downie, J.R.M. Annand, I.J.D. MacGregor (Spokespersons).

[8] P. A. M. Guichon, G. Q. Liu, A. W. Thomas, Nucl. Phys. A 591606 -638 (1995).

[9] D. Drechsel et al., Phys. Rev. C 57,2, 941 (1998).

[10] B. Pasquini et al., Eur. Phys. J. A 11, 185 - 208 (2001).

[11] MAMI Proposal A1-01/09, H. Fonvielle, H. Merkel (Spokespersons).

[12] L. Doria, PhD-Thesis, Mainz 2007, http://wwwa1.kph.uni-mainz.de/A1/publications/doctor/. 
[13] H. B. van den Brink et al., Phys. Rev. Lett. 74 (1995) 3561.

[14] M. O. Distler et al., Phys. Rev. Lett. 80 (1998) 2294.

[15] H. Merkel et al., Phys. Rev. Lett. 88 (2002) 012301.

[16] V. Bernard, N. Kaiser, U.-G.Meißner, Phys. Lett. B 378, 337 (1996).

[17] D. Drechsel et al., Nucl. Phys. A645 (1999) 145-174, 2007 fit.

[18] S.S. Kamalov et al., Phys. Rev. Lett. 83, (1999) 4494; Phys. Rev. C 64 (2001) 032201. 